\title{
Hábitos e Perturbações do Sono numa População Pediátrica
}

\section{Sleep Habits and Disorders in a Pediatric Population}

Diana Rita Oliveira ${ }^{1 *}$, Cristiana Amorim de Carvalho², Susana Oliveira ${ }^{3}$

*Autor Correspondente/Corresponding Author: Diana Rita Oliveira [diana.oliveira@hb.min-saude.pt] Serviço de Pediatria do Hospital de Braga Sete Fontes - São Victor, 4710-243 Braga, Portugal ORCID iD: 0000-0002-3186-6638

\section{RESUMO}

INTRODUÇÃO: O sono tem um papel crucial no desenvolvimento da criança e adolescente. Os distúrbios do sono constituem uma das perturbações comportamentais mais comuns em idade pediátrica. Afetam 20\%-30\% das crianças sendo os mais comuns a insónia comportamental da infância. O Questionário de Hábitos de Sono das Crianças (CSHQ) avalia o sono das crianças em idade escolar, validado em português para aplicação dos 2-10 anos. Este estudo pretendeu caracterizar os hábitos e perturbações do sono numa população pediátrica saudável numa USF e verificar a utilidade e importância do CSHQ-PT.

MATERIAL E MÉTODOS: Estudo descritivo correlacional e transversal numa amostra pediátrica entre os 2-10 anos de idade. Aplicação do CSHQ-PT. Índice de Perturbação do Sono (IPS) com ponto de corte de 44. Amostra selecionada por conveniência. Questionários aplicados durante 3 meses no momento que antecedeu a consulta de Saúde Infantil e Juvenil.

RESULTADOS: Obtiveram-se 157 questionários. IPS acima do ponto de corte em 67,3\%, valores mais elevados nos mais jovens e quando a perceção parental é positiva. Subescalas com valores mais elevados nas parassónias para os rapazes; resistência em ir para a cama, ansiedade no sono e despertares noturnos nas crianças mais novas. Correlação positiva entre despertar noturno e os minutos dormidos à semana.

DISCUSSÃO/CONCLUSÃO: Incidência elevada de perturbações do sono, semelhante ao publicado anteriormente. Mediana de horas de sono concordante com o recomendado pela National Sleep Foundation. Valores de IPS superiores na idade pré-escolar. Destaca-se a disparidade entre a identificação parental e a existência de uma perturbação do sono.

PALAVRAS-CHAVE: Criança; Distúrbios do Início e da Manutenção do Sono; Inquéritos e Questionários; Sono 


\section{ABSTRACT}

INTRODUCTION: Sleep plays a crucial role in the development of children and adolescents. Sleep disorders are one of the most common behavioral disorders in pediatric age. It affects 20\%-30\% of children, the most common being childhood behavioral insomnia. The Children Sleep Habits Questionnaire (CSHQ) evaluates the sleep of school-aged children, validated in Portuguese for application from 2-10 years-old. This study aimed to characterize sleep habits and disorders in a healthy pediatric population in Health Center in the city of Braga and to verify the usefulness and importance of CSHQ-PT.

MATERIAL AND METHODS: Descriptive correlational and cross-sectional study in a sample of children aged 2-10 years using the CSHQ-PT. Sleep Disorder Index (SDI) with a cut-off value of 44. Sample selected by convenience. Questionnaires applied during 3 months before the Child and Youth Health consultation.

RESULTS: A total of 157 questionnaires were filed. SDI above the cutoff value in it was found that 67.3\%, with higher values in the younger age group and when parental perception is positive. Subscales with higher values in parasomnias for males, and in resistance to going to bed, anxiety in sleep and night awakenings in younger children. Positive correlation between night awakenings and the number of minutes slept during weekdays.

DISCUSSION/CONCLUSION: High incidence of sleep disorders, like results previously published. Median of sleeping hours in agreement with the recommended by the National Sleep Foundation. Higher SDI values at preschool age. The disparity between parental identification and the existence of a sleep disorder stands out.

KEYWORDS: Child; Sleep; Sleep Initiation and Maintenance Disorders; Surveys and Questionnaires

\section{INTRODUÇÃO}

sono tem um papel crucial no desenvolvimento da criança e adolescente, com impacto no crescimento, desenvolvimento emocional e comportamental, mas também influencia as suas funções cognitivas, de atenção e aprendizagem, pelo que se assume que os distúrbios do sono podem ter repercussões em todas estas áreas. ${ }^{1}$ Torna-se assim importante o reconhecimento atempado destes distúrbios bem como o seu tratamento, permitindo que a criança seja mais saudável e adquira maior capacidade de aprendizagem.

Os distúrbios do sono constituem uma das perturbações comportamentais mais comuns em idade pediátrica e estima-se que afetem 20\%-30\% das crianças. ${ }^{2} \mathrm{Ne}$ les estão incluídas a insónia comportamental da infância e as parassónias tais como: terrores noturnos, pesadelos, entre outros. ${ }^{3}$ Nas crianças, os distúrbios relacionados com o início do sono e os despertares noturnos são dominantes, presentes em 20\%-25\%.² Estima-se que 40\%-80\% das crianças com distúrbios do sono aos 1548 meses apresentam distúrbios do sono persistentes após 2-3 anos. ${ }^{2}$

Os distúrbios do sono são ainda uma patologia subvalorizada, mesmo por parte dos pais, levando por vezes a dificuldades no diagnóstico por parte dos profissionais de saúde.
Para avaliação das componentes comportamentais e fisiológicas do sono os questionários de preenchimento parental tornaram-se uma importante ferramenta, permitindo a avaliação retrospetiva dos hábitos, perturbações e comportamentos num determinado espaço de tempo. Nesse sentido, foi desenvolvido nos Estados Unidos da América o CSHQ, um questionário retrospetivo para avaliação do sono nas crianças em idade escolar, que tem vindo a ser utilizado em diversos estudos. A sua estrutura baseia-se nos sintomas comuns aos diagnósticos de perturbações do sono mais prevalentes na idade pediátrica de acordo com a Classificação Internacional dos Distúrbios do Sono (ICSD). É composto por 45 itens, agrupados em oito subescalas, cuja soma corresponde ao IPS. Permite avaliar a perceção dos pais do sono dos seus filhos numa semana típica, sugerindo um corte da pontuação total de 44 para identificar as crianças com possíveis distúrbios do sono. A adaptação e validação do CSHQ já estão publicadas em várias línguas, tendo a tradução e adaptação à língua portuguesa (CSHQ-PT) sido publicada em 2011 com aplicabilidade e validação para a população dos 2 aos 10 anos. $^{4}$

Uma vez que as perturbações do sono têm um papel relevante na população pediátrica, e sendo a literatura relativa ao tema ainda limitada, o questionário CSHQ-PT tornou-se um importante instrumento de relevância epidemiológica. 
Este estudo pretendeu caracterizar os hábitos e perturbações do sono numa população pediátrica saudável em seguimento numa Unidade de Saúde Familiar (USF) da cidade de Braga e verificar a utilidade e importância do CSHQ-PT na caracterização dos hábitos e perturbações do sono.

\section{MATERIAL E MÉTODOS}

Estudo descritivo correlacional e transversal numa amostra de cuidadores das crianças utentes da USF Gualtar com idades compreendidas entre os 2 e os 10 anos de idade, através da aplicação do CSHQ-PT validado.

A amostra foi selecionada por conveniência junto da população de utentes da USF com idades entre os 2 e os 10 anos, com um total de 1603 utentes. Para obtenção de uma amostra significativa foi utilizado o programa Survey System ${ }^{\circledR}$, tendo-se obtido um $n=310$ para um nível de confiança de $95 \%$ e definido um $p=0,05$. A este valor acresce-se $10 \%$ de perdas estimadas, perfazendo um total de 341 utentes.

Foram definidos como critérios de exclusão a não-aceitação de participação no estudo, o não preenchimento do consentimento informado, crianças portadoras de doença respiratória com necessidade de ventilação não invasiva durante o sono, crianças com atraso do desenvolvimento psicomotor ou doença do foro psiquiátrico ou com medicação que tem influência no sono, e crianças não residentes em Portugal.

Os questionários foram aplicados no período entre outubro e dezembro de 2018, no momento que antecedeu a consulta de Saúde Infantil e Juvenil, com oportunidade para esclarecimento de dúvidas junto do Médico durante a consulta. O ponto de corte utilizado para identificação das crianças com perturbação do sono foi de 44 .
Análise estatística dos dados através do software Statistical Package for the Social Science ${ }^{\circledR}$ (SPSS ${ }^{\circledR}$ ). Foi analisada a normalidade das variáveis contínuas, sendo que em nenhum dos casos se verificou a normalidade dos dados, pelo que os resultados são apresentados como mediana [amplitude interquartil]. Na análise das subescalas também não se verificou a distribuição normal das variáveis, mantendo-se, contudo, a apresentação dos valores com média e desvio padrão para comparação com outros estudos. Os valores qualitativos são apresentados como frequência absoluta e percentagem. A comparação entre grupos foi analisada através do T-test ou Mann-Whitney para variáveis contínuas entre grupos. Para a análise de correlação foram testadas as variáveis através do teste de Spearman ou do Pearson. Foi definido um valor de significância $p<0,05$.

\section{RESULTADOS}

No período do estudo foram obtidos um total de 157 questionários. A distribuição por género foi relativamente homogénea, com $55,1 \%$ do sexo masculino e $44,9 \%$ sexo feminino. A mediana de idades foi de 5 anos [3;8], com idade mínima de 2 anos e máxima de 10 anos. No sexo feminino a mediana de idades foi de 6 anos e no sexo masculino 5 anos, sem significado estatístico (Tabela 1).

Considerando 44 como ponte de corte na escala IPS, verificamos que $70,9 \%$ do sexo masculino ( $n=61$ ) e $63,8 \%$ do sexo feminino $(n=44)$ se encontram acima deste valor ( $p=0,073$ ), correspondendo a um total de 67,3\% da amostra. Por grupo etário verificamos que $75,90 \%$ das crianças entre os $2-5$ anos de idade e 58,3\% das crianças com 6-10 anos de idade apresentam um IPS acima desse valor. Destaca-se que apenas em 25 casos houve perceção parental da existência de distúrbio do sono, res-

TABELA 1. Diferença por género.

\begin{tabular}{|c|c|c|c|c|c|}
\hline & Categoria & Total & $\begin{array}{c}\text { Masculino } \\
\mathrm{n}=86(55,1 \%)\end{array}$ & $\begin{array}{c}\text { Feminino } \\
\mathrm{n}=70(44,9 \%)\end{array}$ & $p$-value \\
\hline Idade & & $\begin{array}{l}5[3 ; 8] \\
{[2 ; 10]}\end{array}$ & $\begin{array}{l}5[3 ; 8] \\
{[2 ; 10]}\end{array}$ & $\begin{array}{l}6[3 ; 8] \\
{[2 ; 10]}\end{array}$ & 0,373 \\
\hline Minutos dormidos (semana) & & $\begin{array}{c}610[595 ; 630] \\
{[450 ; 705]}\end{array}$ & $\begin{array}{c}615[600 ; 630] \\
{[450 ; 690]}\end{array}$ & $\begin{array}{c}600[587.5 ; 640] \\
{[510 ; 705]}\end{array}$ & 0,580 \\
\hline Minutos dormidos (fim-de-semana) & & $\begin{array}{c}630[600 ; 660] \\
{[450 ; 780]}\end{array}$ & $\begin{array}{c}630[600 ; 660] \\
{[450 ; 780]}\end{array}$ & $\begin{array}{c}660[600 ; 660] \\
{[540 ; 750]}\end{array}$ & 0,166 \\
\hline IPS & & $\begin{array}{c}48[43 ; 53] \\
{[35 ; 75]}\end{array}$ & $\begin{array}{c}49[43 ; 55] \\
{[35 ; 75]}\end{array}$ & $\begin{array}{c}46[43 ; 52] \\
{[35 ; 69]}\end{array}$ & 0,073 \\
\hline Tempo acordado de noite & $\begin{array}{c}<5 \\
5-15 \\
>15\end{array}$ & $\begin{array}{l}26(25,7 \%) \\
63(62,4 \%) \\
12(11,9 \%)\end{array}$ & $\begin{array}{c}16(29,6 \%) \\
29(53,7 \%) \\
9(16,7 \%)\end{array}$ & $\begin{array}{c}10(21,3 \%) \\
34(72,3 \%) \\
3(6,4 \%)\end{array}$ & 0,115 \\
\hline Perceção parental & $\begin{array}{l}\text { Sim } \\
\text { Não }\end{array}$ & $\begin{array}{c}25(16 \%) \\
131(84 \%)\end{array}$ & $\begin{array}{l}17(19,8 \%) \\
69(80,2 \%)\end{array}$ & $\begin{array}{l}8(11,4 \%) \\
62(88,6 \%)\end{array}$ & 0,158 \\
\hline
\end{tabular}

Valores apresentados em mediana [AIQ], [mínimo; máximo] 
tantes 131 com resposta negativa a esta questão, sem diferenças estatisticamente significativas entre sexos ( $p=0,115)$. A mediana do Índice de Perturbação do Sono (IPS), foi de 48 [43;53], sem diferença estatisticamente significativa entre sexos ( $p=0,07)$. Por grupo etário obtivemos uma mediana de IPS de 50 [45;55] para crianças com idades 2-5 anos e de 46 [41;52] para idades 6-10 anos, com significado estatístico $(p=0,07)$ (Tabela 2). Constatamos ainda que o IPS é diferente entre os pais que têm perceção dos filhos apresentarem ou não este problema, com valor do IPS superior quando a perceção parental é positiva $(p<0,001)$.

Relativamente à duração do sono, constatou-se uma mediana de 610 minutos [595;630] durante a semana e de 630 minutos [600-660] ao fim-de-semana, sem diferença estatisticamente significativa entre géneros. Por grupo etário constatou-se uma duração mediana durante a semana de 615 minutos [600;637,5] para crianças entre os 2-5 anos e 600 minutos [570;63] dos 6-10 anos, diferença estatisticamente significativa ( $p=0,002)$. Ao fim-se-semana constatou-se uma duração mediana de 660 minutos [600;690] e 630 minutos [600;660] para os mesmos grupos etários, sem significado estatístico ( $p=0,183)$. Maioria dos despertares noturnos com duração entre 5-15 minutos (62,4\%), apenas 11,9\% referiram despertar noturno superior a 15 minutos, sem diferença estatisticamente significativa entre sexos e entre grupo etário (Tabelas 1 e 2).

TABELA 2. Diferença por grupo etário.

\begin{tabular}{|c|c|c|c|c|}
\hline & Categoria & Entre 2 e 5 anos & Superior a 5 anos & $p$-value \\
\hline Minutos dormidos (semana) & & $\begin{array}{c}615[600 ; 637,5] \\
{[450 ; 705]}\end{array}$ & $\begin{array}{c}600[570 ; 630] \\
{[505 ; 690]}\end{array}$ & 0,002 \\
\hline Minutos dormidos (fim-de-semana) & & $\begin{array}{c}660[600 ; 690] \\
{[450 ; 780]}\end{array}$ & $\begin{array}{c}630[600 ; 660] \\
{[540 ; 780]}\end{array}$ & 0,183 \\
\hline IPS & & $\begin{array}{c}50[45 ; 55] \\
{[35 ; 69]}\end{array}$ & $\begin{array}{c}46[41 ; 52] \\
{[35 ; 75]}\end{array}$ & 0,007 \\
\hline Tempo acordada de noite & $\begin{array}{l}<5 \\
5-15 \\
>15\end{array}$ & $\begin{array}{c}13(25 \%) \\
32(61,5 \%) \\
7(13,5 \%)\end{array}$ & $\begin{array}{c}13(26,5 \%) \\
31(63,3 \%) \\
5(10,2 \%)\end{array}$ & 0,878 \\
\hline Perceção parental & $\begin{array}{l}\text { Sim } \\
\text { Não }\end{array}$ & $\begin{array}{l}15(18,3 \%) \\
67(81,7 \%)\end{array}$ & $\begin{array}{l}10(13,5 \%) \\
64(86,5 \%)\end{array}$ & 0,416 \\
\hline
\end{tabular}

Valores apresentados em mediana [AIQ], [mínimo; máximo]

TABELA 3. Avaliação das subescalas e diferenças entre género.

\begin{tabular}{|c|c|c|c|c|}
\hline & Total & Masculino & Feminino & $p$-value \\
\hline Parassónias & $5,55 \pm 1,52$ & $5,85 \pm 1,72$ & $5,18 \pm 1,14$ & 0,014 \\
\hline Resistência em ir para a cama & $9,69 \pm 3,1$ & $10,03 \pm 3,16$ & $9,26 \pm 3$ & 0,120 \\
\hline Despertar noturno & $4,28 \pm 1,53$ & $4,42 \pm 1,62$ & $4,1 \pm 1,42$ & 0,198 \\
\hline Sonolência diurna & $13,74 \pm 2,78$ & $13,77 \pm 2,83$ & $13,71 \pm 2,73$ & 0,906 \\
\hline Distúrbios respiratórios & $3,43 \pm 1,05$ & $3,42 \pm 1,11$ & $3,44 \pm 0,97$ & 0,886 \\
\hline Ansiedade no sono & $6,97 \pm 2,32$ & $7,07 \pm 2,34$ & $6,86 \pm 2,32$ & 0,571 \\
\hline
\end{tabular}

Valores apresentados em média \pm DP

TABELA 4. Avaliação das subescalas e diferenças entre grupo etário.

\begin{tabular}{|c|c|c|c|c|}
\hline & Total & $\leq 5$ anos & $>5$ anos & $p$-value \\
\hline Parassónias & $5,55 \pm 1,52$ & $5,76 \pm 1,51$ & $5,45 \pm 1,52$ & 0,297 \\
\hline Resistência em ir para a cama & $9,69 \pm 3,1$ & $10,39 \pm 3,04$ & $8,91 \pm 3$ & 0,003 \\
\hline Despertar noturno & $4,28 \pm 1,53$ & $4,82 \pm 1,72$ & $3,68 \pm 1,01$ & $<0,001$ \\
\hline Sonolência diurna & $13,74 \pm 2,78$ & $13,74 \pm 2,91$ & $13,74 \pm 2,64$ & 0,999 \\
\hline Distúrbios respiratórios & $3,43 \pm 1,05$ & $3,49 \pm 1,12$ & $3,36 \pm 0,96$ & 0,466 \\
\hline Ansiedade no sono & $6,97 \pm 2,32$ & $7,39 \pm 2,22$ & $6,51 \pm 2,36$ & 0,018 \\
\hline
\end{tabular}

Valores apresentados em média 土DP 
TABELA 5. Relação qualidade e quantidade de sono.

\begin{tabular}{l|c|c|}
\hline & Minutos dormidos semana & Minutos dormidos fim-de-semana \\
\hline Parassónias & $0,693[0,038]$ & $0,273[-0,105]$ \\
\hline Resistência em ir para a cama & $0,155[0,115]]$ & $0,931[-0,007]$ \\
\hline Despertares noturnos & $0,016[0,195]$ & $0,924[-0,008]$ \\
\hline Sonolência diurna & $0,193[-0,105]$ & $0,644[-0,038]$ \\
\hline Distúrbios respiratórios do sono & $0,466[-0,059]$ & $0,369[-0,073]$ \\
\hline Ansiedade relacionada com o sono & $0,077[0,143]$ & $0,737[-0,027]$
\end{tabular}

Os valores na tabela referem-se a p-value [Rhô]

TABELA 6. Comparação dos dados do estudo atual com os dados do estudo publicado em 2014.

\begin{tabular}{l|c|c} 
& $\begin{array}{c}\text { Estudo atual } \\
(2018)\end{array}$ & Estudo Ata Pediátrica (2014) \\
\hline Parassónias & $5,55 \pm 1,52$ & $8,82 \pm 1,84$ \\
\hline Resistência em ir para a cama & $9,69 \pm 3,1$ & $7,98 \pm 2,54$ \\
\hline Despertares noturnos & $4,28 \pm 1,53$ & $3,87 \pm 1,18$ \\
\hline Sonolência diurna & $13,74 \pm 2,78$ & $13,98 \pm 2,6$ \\
\hline Distúrbios respiratórios relacionados com o sono & $3,43 \pm 1,05$ & $3,51 \pm 1,0$ \\
\hline Ansiedade relacionada com o sono & $6,97 \pm 2,32$ & $5,56 \pm 1,88$
\end{tabular}

Dados apresentados em média \pm DP

Na avaliação das subescalas, foram encontradas diferenças estatisticamente significativas entre sexos para as parassónias, com médias mais elevadas no sexo masculino ( $p=0,014)$, e entre grupos etários com maiores valores no grupo até aos 5 anos na resistência em ir para a cama $(p=0,003)$, ansiedade no sono $(p=0,018)$ e despertares noturnos $(p<0,001)$. Nas Tabelas 3 e 4 estão representados os valores referentes às subescalas distribuídos por sexo e grupo etário, respetivamente.

Foram ainda relacionadas a quantidade e qualidade do sono, pela comparação do número de horas de sono dormidas à semana e ao fim-de-semana com as subescalas do questionário (Tabela 5). De realçar a correlação positiva do despertar noturno com os minutos dormidos à semana ( $p=0,016$; rho $=0,195)$.

\section{DISCUSSÃO}

Neste estudo obtivemos diferenças significativas na duração do sono à semana entre os grupos etários, verificando-se uma diminuição dos minutos dormidos com o aumento da idade. Esta tendência verificou-se também na duração do sono ao fim-de-semana, contudo sem significado estatístico. Não se encontrou diferença significativa entre géneros, resultados que parecem ser concordantes com o publicado na literatura. Para todos os grupos obtivemos uma mediana de sono de pelo me- nos 10 horas, o tempo recomendado pela National Sleep Foundation (NSF) em idade escolar.

No que diz respeito à qualidade do sono, expressa no IPS, os valores obtidos na generalidade situaram-se acima do ponto de corte, com cerca de 2/3 da amostra a apresentar IPS acima de 44. A diferença do IPS não foi significativa entre sexos, mas sim entre faixas etárias, verificando-se valores superiores na idade pré-escolar (2-5 anos). Esta diferença pode dever-se ao tamanho da amostra com maior número de crianças abaixo de 5 anos (52,6\%) comparativamente a outros estudos realizados. Nas subescalas os valores obtidos foram, em algumas, semelhantes ao publicado no estudo de $2014,{ }^{1}$ com disparidade mais evidente nas parassónias e resistência em ir para a cama (Tabela 6). As diferenças significativas por género observaram-se nas parassónias, com pontuação mais elevada no sexo masculino, também concordante com a literatura. ${ }^{1}$ A comparação dos grupos etários encontrou diferenças significativas na resistência em ir para a cama, ansiedade no sono e despertares noturnos, com o grupo com idade inferior a 5 anos com pontuação superior. Não obtivemos valores mais elevados em nenhuma subescala no grupo etário dos mais velhos, ao contrário do descrito na literatura. Salienta-se ainda a relação positiva entre o despertar noturno e os minutos dormidos à semana. 
Os autores consideram relevante a disparidade encontrada entre a identificação parental da existência de uma perturbação do sono e a identificação de comportamentos sugestivos de acordo com o questionário. Salienta-se que apenas 16,6\% dos pais identificaram a existência de perturbação do sono, valor superior ao encontrado no estudo português de 2014. ${ }^{1}$ Estes valores evidenciam a necessidade de ocorrência de manifestações mais evidentes de disfunção comportamental relacionada com o sono para que seja identificada pelos pais. Não podemos, contudo, excluir a possibilidade de os resultados serem devidos à não compreensão das questões pelos pais devido ao baixo nível social e intelectual, parâmetro não contemplado na implementação deste questionário. Este questionário é um instrumento útil na avaliação médica das crianças e para um rastreio e diagnóstico de perturbações do sono desde que corretamente aplicado.

Tal como já questionado no estudo de 2014, também os autores questionam a pontuação utilizada para ponto de corte do IPS e a sua adequação à amostra utilizada.

É importante o desenvolvimento de estudos multicêntricos para aferição de resultados futuros da aplicabilidade do CSHQ-PT na prática clínica. Este trabalho caracteriza uma população pediátrica numa área da saúde que é fundamental, e realça a importância que os instrumentos psicométricos validades desempenham na avaliação de crianças com possíveis perturbações do sono.

Como pontos negativos do estudo destacamos a não representatividade da amostra para a população selecionada que não nos permite tirar conclusões ou significância estatística para a população geral. Como limitações destacamos a ausência de informação relativa ao nível social e intelectual dos pais que poderiam elucidar alguns dos resultados obtidos, uma vez que pode ter influência no correto preenchimento das questões, bem como nos comportamentos das crianças.

\section{CONCLUSÃO}

Os autores concluem que a amostra estudada apresenta uma elevada incidência de perturbações do sono, resultados que parecem semelhantes ao publicado anteriormente em Portugal. A diferença obtida em alguns parâmetros nos diferentes grupos etários depreende que as perturbações podem também variar de acordo com as etapas do desenvolvimento da criança. $\bigcirc$ sono desempenha um importante papel para o bem-estar geral da criança e do adolescente, com impacto no comportamento e desempenho escolar, mas também na saúde.
Assim, a avaliação dos hábitos de sono deve ser contemplada nas consultas de saúde infantil, com especial destaque quando reportada a existência de um problema por parte dos pais. A educação parental desempenha um importante papel na identificação dos distúrbios do sono, permitindo o reconhecimento atempado e melhoria na adesão aos planos terapêuticos comportamentais com recurso à farmacoterapia apenas quando justificada.

\section{RESPONSABILIDADES ÉTICAS}

CONFLITOS DE INTERESSE: Os autores declaram a inexistência de conflitos de interesse na realização do presente trabalho.

FONTES DE FINANCIAMENTO: Não existiram fontes externas de financiamento para a realização deste artigo.

CONFIDENCIALIDADE DOS DADOS: Os autores declaram ter seguido os protocolos da sua instituição acerca da publicação dos dados de doentes.

PROTEÇÃO DE PESSOAS E ANIMAIS: Os autores declaram que os procedimentos seguidos estavam de acordo com os regulamentos estabelecidos pelos responsáveis da Comissão de Investigação Clínica e Ética e de acordo com a Declaração de Helsínquia de 2013 da Associação Médica Mundial.

PROVENIÊNCIA E REVISÃO POR PARES: Não comissionado; revisão externa por pares.

\section{ETHICAL DISCLOSURES}

CONFLICTS OF INTEREST: The authors have no conflicts of interest to declare.

FINANCING SUPPORT: This work has not received any contribution, grant or scholarship.

CONFIDENTIALITY OF DATA: The authors declare that they have followed the protocols of their work center on the publication of data from patients.

PROTECTION OF HUMAN AND ANIMAL SUBJECTS: The authors declare that the procedures were followed according to the regulations established by the Clinical Research and Ethics Committee and to the 2013 Helsinki Declaration of the World Medical Association.

PROVENANCE AND PEER REVIEW: Not commissioned; externally peer reviewed. 


\section{REFERÊNCIAS}

1. Arriaga C, Brito S, Gaspar P, Luz A. Hábitos e Perturbações do Sono: Caracterização de uma Amostra Pediátrica na Comunidade. Acta Pediatr Port. 2015;46:367-75.

2. Bruni $O$, Novelli L. Child health Sleep disorders in children. BMJ Clin Evid. 2010;2010:2304.

3. Monteiro T. Perturbações do sono na criança perspectiva do pediatra. Nascer Crescer. 2006;15:180-3.

4. Silva FG, Silva CR, Braga LB, Neto AS. Portuguese Children's Sleep Habits Questionnaire - Validation and cross-cultural comparison. J Pediatr. 2014;90:78-84. doi:10.1016/j.jpedp.2013.06.008. 